\title{
Kulturwandel im Krankenhaus
}

\author{
Wie die Digitalisierung die Arbeit verändert
}

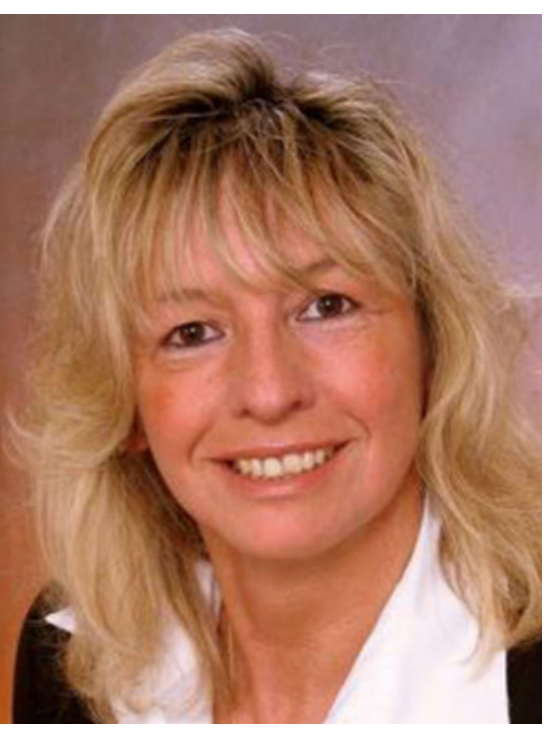

Dr. Gabriele Angenendt, psychologische Psychotherapeutin, ist seit 25 Jahren in verschiedenen Funktionen in der Patientenbehandlung tätig, zudem 10 Jahre im Management. Von 2010 bis Ende 2016 war sie Leitende Psychologin und stellvertretende Direktorin in der salus klinik Hürth in Köln. Die Fachklinik für Abhängigkeitserkrankungen und Psychosomatik bietet ein multimodales Behandlungskonzept mit medizinischen, therapeutischen und sozialen Bausteinen. Bedarf und Nachfrage steigen. Ab Juni 2017 werden die Behandlungskapazitäten um 80 auf 320 Betten aufgestockt. Die Patienten bleiben in der Regel 3-5 Wochen, bei Sucherkrankungen können bis zu 26 Wochen erforderlich sein. Die Dokumentation der Patientendaten erfolgt IT-gestützt. Die Informationen werden in einer Datenbank zentral gespeichert und stehen den autorisierten Mitarbeitern über ein PC-Netzwerk zur Verfügung, sodass die Leistungsangebote patientenindividuell aufeinander abgestimmt werden können. Ferner kommen Apps im Betrieblichen Gesundheitsmanagement zum Einsatz.

Frau Dr. Angenendt, welche Rolle spielt die Informationstechnologie in der sprechenden Medizin?
Die Verwaltungsabläufe laufen wie in der somatischen Medizin IT-gestützt. Ebenso beginnen Kliniken ihre Behandlungsdokumentation auf eine digitale Patientenakte umzustellen. Für eine optimale Zusammenarbeit müssen alle Fachgruppen auf die Informationen zugreifen können. Nur so können valide Schlüsse gezogen werden. Großer Nachholbedarf besteht noch bei der intersektoralen Vernetzung. Derzeit verzögert sich die Behandlung oft, weil der Patient bei der Aufnahme nur unvollständige Unterlagen beibringt. Es wäre wesentlich einfacher, wenn uns die Informationen der Arztpraxis oder des Akutkrankenhauses digital schon bei Aufnahme vorlägen. Dafür fehlen aber noch die rechtlichen und sicherheitstechnischen Voraussetzungen.

\section{Gibt es Einsatzfelder für telemedizini- sche Anwendungen?}

In Deutschland gibt es kaum Beispiele für Digital Health in der sprechenden Medizin. Die AOK Nordost erprobt derzeit Möglichkeiten der digitalen Psychotherapie. Hierbei finden Diagnostik und Therapieplanung wie gewohnt in der Praxis statt. Die therapeutischen Sitzungen laufen anschließend per Videokonferenz. Digitale Medien ließen sich meines Erachtens auch bei der Katamnese von Suchterkrankten sinnvoll einsetzen, um schneller Information über einen möglichen Rückfall zu erhalten und unterstützen zu können. Natürlich besteht auch der Einwand, dass die Psychotherapie eine tragfähige Beziehung erfordert, für die der persönliche Kontakt von Arzt und Patient essenziell ist. Andererseits zeigen Studien aus anderen Ländern, dass die Wirksamkeit und Nachhaltigkeit der Internettherapie mit der klassischen Therapie vergleichbar ist. Die Niederlande, Schweden und Australien sind auf diesem Gebiet weiter als wir.

\section{Was bedeutet die Digitalisierung für den beruflichen Alltag der Ärzte und Therapeuten?}

Die Digitalisierung bringt nicht nur neue Arbeitsinhalte mit sich, sondern bedingt ein neues berufliches Selbstverständnis, da die bestehenden Strukturen aufbrechen.
Ärzte und Therapeuten werden sich ein stückweit zurückzunehmen müssen und lernen, sich als ein „Tool“ neben anderen Behandlungsmöglichkeiten zu verstehen. Technische Assistenz wird nötig sein, um das rasant anwachsende Wissen in der Medizin tatsächlich verarbeiten zu können. Da die Patienten nach den bestmöglichen Behandlungsbedingungen suchen, werden sie in ihre Entscheidung für oder gegen ein Haus einbeziehen, ob die Fachkräfte und Einrichtungen hierauf eingerichtet sind oder nicht.

\section{Inwieweit verändern sich die Arbeits- prozesse? Welche Kompetenzen gewinnen an Bedeutung?}

Es wird flexibler werden. Wenn indikationsoder themenbezogene Videokonferenzen in das therapeutische Konzept einer Klinik integriert werden, können Therapeuten einen Teil ihrer Arbeit standortungebunden leisten und Fahrtzeit sparen. Außerdem wird es leichter, externe Spezialisten in die Arbeit einzubeziehen, die für mehrere Kliniken parallel tätig sind. Auch die Übergaben lassen sich digital gestalten, wodurch die starren Arbeitszeitmodelle auf den Stationen ihre Notwendigkeit verlieren. Die Krankenhäuser müssen aber hierfür erst noch den Freiraum schaffen. Perspektivisch gewinnen projektbezogene Arbeiten an Bedeutung, auch im Klinikbetrieb. Die Mitarbeiter werden dadurch weniger abhängig von ihrem direkten Vorgesetzten, wenn sie ihren nächsten Karriereschritt planen. Gefragt sind die Fähigkeiten zur Selbstführung und Selbststrukturierung. Dies gilt besonders für Fachkräfte, die weniger eng an ein Haus gebunden sind. Hinzu kommen Kreativität und die Bereitschaft sich neuen Anforderungen zu stellen.

\section{Schlägt sich dies in der Personalfüh- rung nieder?}

Heute basiert Führung im Klinikbetrieb auf Macht und Weisungsbefugnissen. Künftig wird es mehr darum gehen, Ressourcen und Wissen aus verschiedenen Quellen bereitzustellen, Entscheidungsspielräume zuzulassen und die Mitarbeiter so zu fördern, 
dass sie ihre Kompetenzen kontinuierlich weiterentwickeln und einbringen können. Dies erfordert ein Umdenken auf der Führungsebene. Das heißt, Führungskräfte müssen ihren alten Führungsstil hinterfragen und lernen, Vertrauen in die Fähigkeiten ihrer Mitarbeiter zu entwickeln. Da die Arbeitsformen flexibler werden, wird das Führen auf Distanz zu einem Erfolgsfaktor im Wettbewerb der Krankenhäuser. Die eigene Rolle in der Medizin und als Führungskraft neu zu definieren, ist aber wohl die größte Herausforderung, vor der die Digitalisierung noch steht.

Wie kann ein Krankenhaus digitale Techniken so einsetzen, dass sie von den Beschäftigten positiv wahrgenommen werden?

Zunächst gilt es zu verstehen, dass mit jeder Veränderung Ängste verbunden sind. Menschen halten gern an erprobten Strukturen fest. Das gilt für ältere noch eher als für die jungen. Etwas grundlegend Neues wie die Digitalisierung des Arbeitsprozesses führt leicht zur Verunsicherung. Selbst wenn die Vorteile gesehen werden, befürchten die meisten, etwas falsch machen zu können. Fehler passieren, doch dürfen sie nicht verschwiegen werden. Um aus Fehlern lernen zu können, sind Transparenz und Nachsicht wichtig. Eigene Lernerfahrungen mit Apps oder anderen Teleinstrumenten können helfen, den Nutzen zu erkennen. In der salus Klinik Hürth haben wir zum Beispiel einen Schrittzähler in ein Projekt des Betrieblichen Gesundheitsmanagements integriert.

\section{Welche Bedeutung messen Sie der Digitalisierung für Ihre Arbeit als Psychotherapeutin bei?}

Die Entwicklung zur Telemedizin bzw. Teletherapie ist aus der Psychotherapie nicht mehr wegzudenken. Dennoch wird es noch viele ethische Diskussionen geben, wie die einzelnen Instrumente sinnvoll eingesetzt und die Arzt-Patienten-Kommunikation geregelt werden kann. Selbst die Dauer und Indikation für stationäre Aufenthalte könnte zur Diskussion stehen. Genauso wichtig ist es, einen neuen Dialog mit der Selbsthilfe zu finden, denn auch hier verändert Digital Health die Strukturen. Statt in klassischen Selbsthilfegruppen, deren Mitgliederzahlen rückläufig sind, suchen chronisch Kranke immer häufiger in Sozialen Netzen Unterstützung. Darauf sollten Fachinstitutionen des Gesundheitsbereichs und auch Selbsthilfeorganisationen mit adäquaten Angeboten reagieren.

\section{Vielen Dank für das Gespräch, Frau Dr.} Angenendt!

Das Interview führte Dr. Adelheid Weßling, freie Journalistin, Düsseldorf. 